\title{
TCP Enhancement Using Active Network Based Proxy Transport Service
}

\author{
Siddharth Patil and Mohan Kumar \\ Department of Computer Science and Engineering, \\ The University of Texas at Arlington, Arlington TX 76019, USA, \\ siddharth@utaengineers.com, kumar@cse.uta.edu
}

\begin{abstract}
TCP performance degrades with increasing round trip time $(r t t)$ due to longer slow start periods and slower rate of increase of the congestion window. The proposed, PTS (proxy transport service) is an active network based service, that can improve the end-to-end performance of TCP connections with large $r t t$ 's. PTS also reduces the response time of TCP to congestion and can be used by other active services. A PTS session consists of two separate TCP connections, one between the source host and a designated intermediate node (the PTS node), and another between the PTS node and the destination host. PTS complements TCP without compromising reliability. Simulation studies show the effect of PTS node location, buffer size and node service time on the performance of PTS. Performance can be significantly improved by deploying an interactive distributed service that allows the host to choose the proxy node.
\end{abstract}

\section{Introduction}

In addition to providing reliable communication, Transmission Control Protocol (TCP) effects error control, flow control and resequencing of segments received out of order. TCP uses the arrival rate of acknowledgments to adjust the current size of congestion window (cwnd). During bulk data transfer, the TCP connection frequently alternates between slow start and congestion avoidance phases. The rate at which the cwnd increases depends upon the round trip time $(r t t)$ of TCP segments. Current variants of TCP give good bulk data transfer performance over shorter connection paths. However, as the separation between the source and the destination host increases, $r t t$ increases resulting in reduced TCP throughput. TCP enters slow start phase more frequently for longer connection paths. In this paper we propose, proxy transport service (PTS), an active network based service that improves the TCP bulk data throughput for TCP connections with large average $r t$. The $r t$ for TCP packets on a connection can be large when the connection spans multiple subnets, or when one of the node en route is congested with no alternate route available to the destination or when propagation delay for one of the intermediate links is very high (e.g., a satellite link or a slow wireless link). PTS service is deployable at various active nodes that can be programmed to perform desirable computations on user 
packets within the network. PTS works by splitting the TCP connection between the sender and the receiver into two separate TCP connections: i) PTS Connection-1 between the source host and a chosen active node (called the PTS node); and ii) PTS Connection-2 between the PTS node and the destination host. Ideally, PTS node is an active node located in the path between the source and the destination host such that it is approximately equidistant in terms of delay from each of the hosts. A PTS node acknowledges the source's TCP segments on PTS Connection-1 and forwards the segments to the destination host on PTS Connection-2. PTS node implements all the functionalities of a host TCP sender for PTS Connection-2 i.e., it retransmits lost segments, performs flow control and responds to congestion. PTS throughput for a session depends on three parameters: location of the PTS node, mean time $\left(T_{r}\right)$ an average packet spends inside the PTS node, and the buffer space available at the PTS node. The location of the PTS node determines the rtt for the two PTS connections. Since the PTS node cannot transmit TCP segments as fast as they arrive, large buffer space will be required for this session. Otherwise segments will be dropped at the PTS node leading to poor TCP throughput. As the number of PTS sessions handled by a PTS node increases, $T_{r}$ increases, leading to reduced PTS throughput. PTS complements TCP to improve performance for long delay connections without compromising TCP's reliability. PTS does not reduce the end-to-end delay for a TCP segment but it improves the rate at which the sender's congestion window increases. Through simulation studies we show that PTS improves the TCP throughput for TCP connections having large average $r t t$. Also we show the effect of PTS node location, PTS service time and buffer space per session on PTS throughput. Current research in the areas of security, active node architectures, active packet formats and active network applications will provide the essential components required to deploy PTS [1 23/4].

\subsection{Related Work}

The concept of using a proxy transport service for improving TCP throughput has been in use in wireless networks and in networks with high delay variations. Indirect TCP (I-TCP): In ITCP, the TCP connection between a fixed host $(\mathrm{FH})$ and a mobile host $(\mathrm{MH})$ is broken into two separate TCP connections [5]. The variant of TCP residing in the $\mathrm{MH}$ and the base station (BS) masks effects of mobility. The connection between the FH and the BS uses standard TCP. The BS acknowledges the TCP segments from the FH increasing the TCP throughput of the sender, and transmits the TCP segments over the wireless link. The $\mathrm{BS}$ also intercepts retransmission requests from the $\mathrm{MH}$ and retransmits using a cached copy if available instead of propagating the request all the way to the FH. ITCP can be considered as a specialization of PTS with the BS acting as the PTS node. PTS service can also work over connections spanning heterogeneous networks. An intermediate link can be a satellite link or a wireless last hop. Unlike ITCP, where the location of the proxy is fixed at the BS, PTS provides users with a proxy transport service at a location that can give maximum throughput. In ITCP, the FH is not aware of the proxy service. The nontransparent approach 
adopted by PTS distributes knowledge among the hosts and provides flexibility in selecting a suitable PTS node according to the host requirements.

Performance Enhancement Proxies (PEP): Performance Enhancement Proxies (PEP) proposed in [6] uses transport proxies to improve TCP throughput. PEP residing on the network edge monitors all TCP flows going in and out of the network and manipulates their TCP ACK packets to improve TCP throughput. PEP is a transparent service that has the same problems as that of ITCP with regards to fixed proxy location and transparent service.

QoS using RSVP: RSVP (Resource reservation protocol) is the signaling protocol used for resource reservation in integrated services framework to provide quality of service 7]. RSVP requires a state to be maintained at each and every node in between the sender and the receiver for every single session in order to provide guarantees of delay, bandwidth or packet loss. RSVP does not scale to multiple users. PTS does not require a state like RSVP to be maintained at each and every node en route.

\section{TCP Enhancement}

In this section we discuss the flow control mechanism employed by TCP and the PTS enhancement that improves TCP performance. If the two hosts of a TCP transmission are not local, then packets can get queued at an intermediate router. When a new TCP connection is established the sender initializes its cwnd to 1, and increases cwnd by one whenever an acknowledgement is received. The maximum number of unacknowledged segments that can be sent is equal to the minimum of the advertised window and the cwnd. The bulk data throughput of the TCP connection is affected by flow control and slow start algorithm. The theoretical maximum window size which can give maximum TCP throughput is dependent on the bandwidth-delay product of the connection, given by, capacity (bits) $=$ bandwidth(bits/sec) $* \operatorname{rtt}($ sec $)$. A TCP sender can detect a packet drop either when a retransmission timer(rto) expires or when duplicate ACK's are received. A timeout is a better indication of congestion inside the network. A duplicate ACK indicates out-of-order delivery of packets. When a timeout occurs cwnd is set to 1 and slow start begins. Congestion avoidance is flow control imposed by the sender, while advertised window is flow control imposed by the receiver. Long connection paths result in larger $r t t$ 's. Larger the $r t t$, slower is the rate at which ACK's are received. During slow start the rate at which cwnd increases depends on the rate at which ACK's arrive. For higher $r t t$ 's, it takes much longer for a TCP throughput to reach the network capacity. During congestion avoidance phase, a TCP connection spanning longer paths takes much longer time to increase its throughput. A TCP connection having large number of hops is also quite likely to span across networks under different administrative domains.

In PTS, two separate TCP connections are established, one between the source host and a designated intermediate node (the PTS node), and another between the PTS node and the destination host. All the TCP segments from the 
sending host are acknowledged (to the source) and forwarded by the PTS node (to the destination) as illustrated in Fig. 1.

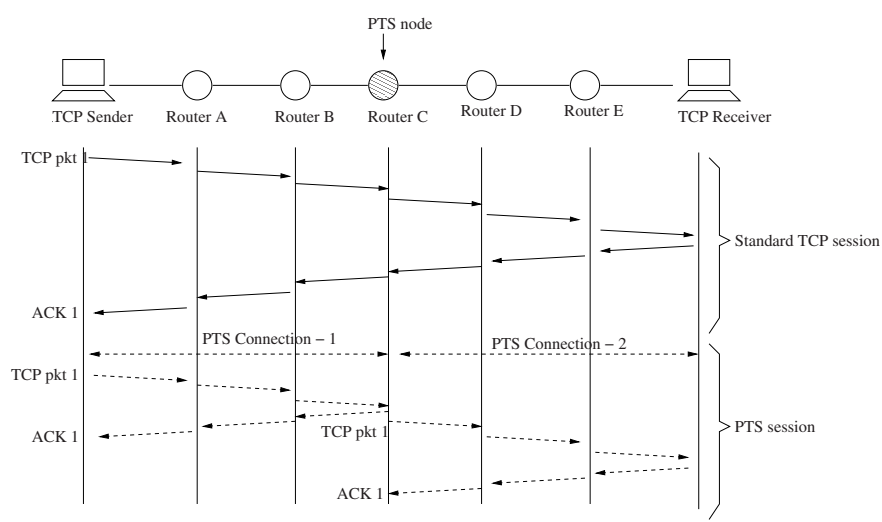

Fig. 1. Proxy Transport Service

During the setup of a PTS session, a requested amount of buffer space is provided for that session inside the PTS node. For every TCP segment received by the PTS node, a copy of the segment is stored in the buffer and an acknowledgment is sent to the source host. PTS node in turn transmits accepted TCP segments to the destination host on PTS Connection-2. The copy of the TCP segment is preserved in the buffer until an acknowledgment is received from the destination host. The PTS node also takes care of retransmitting unacknowledged segments on PTS Connection-2 when a timeout occurs or duplicate ACK's are received.

\section{Simulation Results and Analysis}

This section compares the TCP throughput of PTS with that of a direct TCP connection and other proxy schemes. Network Simulator 2 [8] was used for the simulation studies. The performance of a proxy service is affected by three parameters namely: PTS node location $(X)$, PTS service time $\left(T_{s}\right)$ and PTS buffer capacity per session $\left(C_{\max }\right)$. The simulation topology consists of two hosts separated by variable number of hops $\left(N_{\text {hops }}\right)$. The location of the PTS node is indicated by $X$, which refers to the number of hops between the PTS node and the source host. All the links between the two hosts have the same capacity $\left(L_{C}\right)$ and propagation delay $\left(L_{d}\right)$. Tahoe TCP agents reside on the two hosts. We simulated ftp application traffic between two hosts. PTS functionality is provided in the form of an agent. Any node in between the sender and the receiver can become a PTS node by executing the PTS agent. PTS node uses a single FIFO queue for every session. To represent a more realistic scenario, the service time 
of the PTS node is exponentially distributed over mean service time $T_{s}$. The ftp application sends unlimited packets from the source host to the destination host.

We use fixed sized packets (1000 bytes) throughout the simulation. A DirectTCP and a PTS-connection are compared in terms of cwnd and seqno measured in packets. Also, we use TCP throughput (number of segments received by the destination host per unit time) to measure performance.

\subsection{Comparison of PTS and TCP}

As can be observed from Fig. 2 the slow start period lasts longer for TCP than that for PTS connections due to the reduced $r t t$ for each of the PTS connections. Also the rate of increase in cwnd during congestion avoidance is larger in case of PTS because the ACK's arrive faster. More number of packets are sent in PTS within the same time frame due to reduced $r t t$ for each PTS connection.

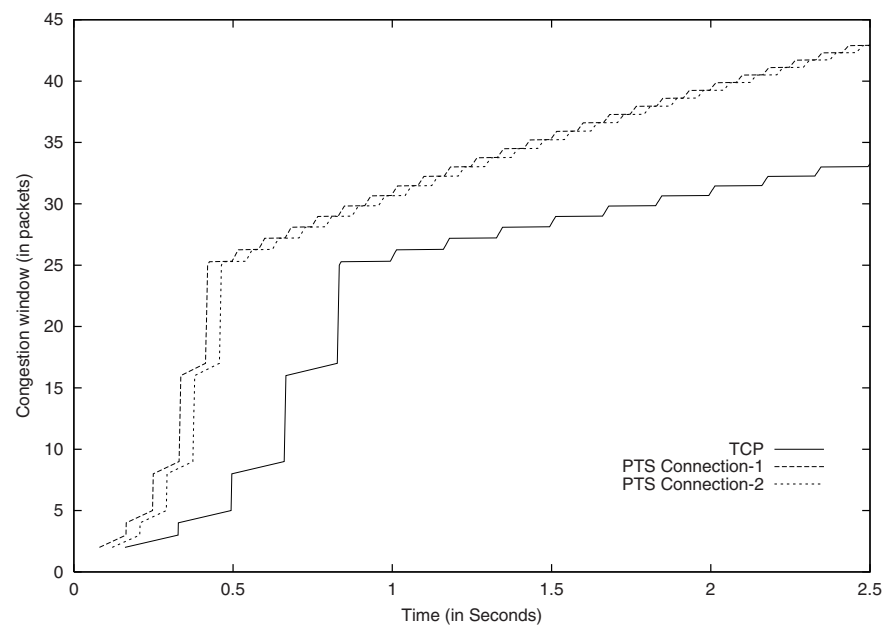

Fig. 2. Congestion Window Comparison for TCP and PTS. $L_{C}=10 M b, L_{d}=$ $10 m s, T_{s}=100 \mu s, N_{\text {hops }}=8, X=4$

The extra delay that a packet suffers within the PTS node overlaps with the transmission of previously processed packets at the node. For the experimental results in Fig. 2 to 4, $L_{C}=10 \mathrm{Mb}, L_{d}=10 \mathrm{~ms}, T_{s}=100 \mathrm{mico} \mathrm{sec}, N_{\text {hops }}=$ 4 and $X=4$. Figure 3 shows the performance of PTS and TCP sessions when timeout occurs or duplicate ACKS are received. Both TCP and PTS sessions are subjected to the same amount of congestion in the same part of the network, at the same time. In this case, the packet drop occurs in between the source host and the PTS node. As soon as the source host detects a possible packet loss, it sets the cwnd to 1 for both TCP and PTS sessions. Because the packet drop 


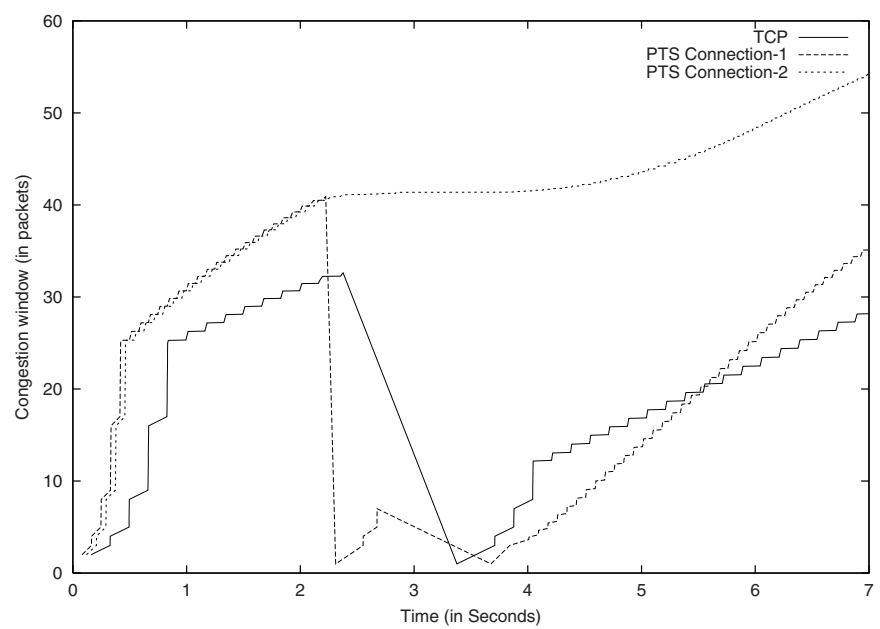

Fig. 3. CWND Comparison of TCP and PTS with Congestion. $L_{C}=10 M b, L_{d}=$ $10 m s, T_{s}=100 \mu s, N_{\text {hops }}=8, X=4$

occurs in between the source and the PTS node, the cwnd of PTS Connection-2 is not affected. PTS node keeps transmitting to the destination host as long as it has unsent segments in its buffer. Thus the end-to-end TCP throughput will not be affected if the congestion in the first part of the PTS connection is momentary. If the congestion were to be in the second part of the PTS connection then the PTS node will set its cwnd to 1 causing the TCP throughput to fall.

If the congestion in the second part of the PTS connection is not momentary then at some point the PTS node is going to run out of buffer space. Due to shorter rtt PTS node will recover its steady state transmission rate for this session much faster than a direct TCP connection. Figure 4 compares the seqno for PTS and TCP sessions. A dip in the plot signifies that no ACKs have been received for segments starting from that seqno and hence TCP is going to retransmit packets. The first dip for both sessions has been marked. PTS detects congestion almost $1 s$ before a direct TCP connection.

PTS Node Location: Location of the PTS node along the path between the source and destination host determines the end-to-end performance of the PTS session as shown in Fig. $5=0$ represents a direct TCP session. As the PTS node location moves towards the center of the path we are able to achieve higher end-to-end reception rates. Assuming that the PEP [6] is located at a fixed location of 6 hops from the sender, we can see why the "one fixed proxy serves all" approach will not be able to give the best throughput to each and every host.

PTS Service Time: Every TCP segment will be delayed by a bounded amount of time inside the PTS node. This time consists of the waiting time to get serviced and the service time to modify the TCP header of the segment. The PTS node modifies the TCP header of accepted segments that arrived on PTS Connection1 from the source host before forwarding them on PTS Connection-2. The packet might be copied within the PTS node more than once before it is queued for 


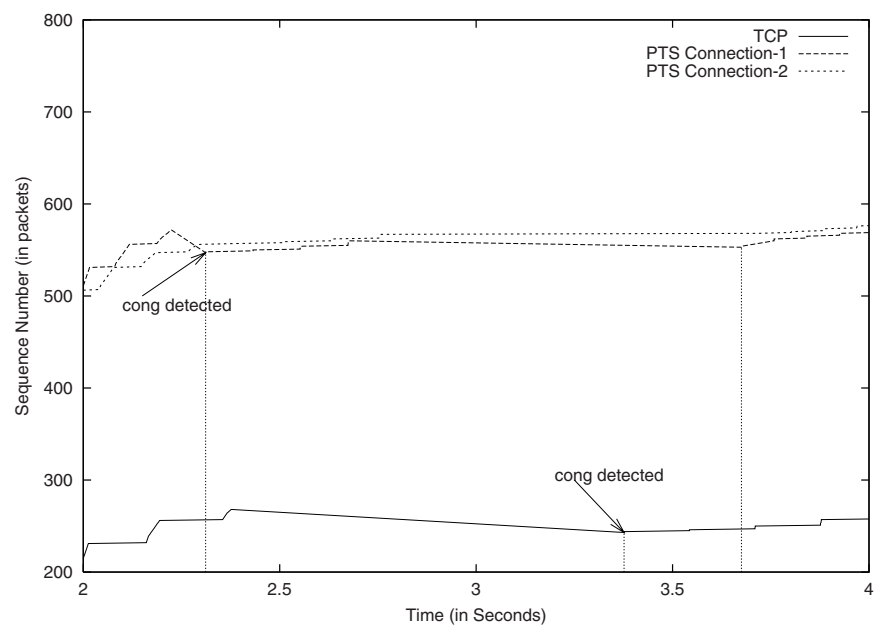

Fig. 4. Seqno Comparison of TCP and PTS with Congestion. $L_{C}=10 M b, L_{d}=$ $10 \mathrm{~ms}, T_{\mathrm{s}}=100 \mu \mathrm{s}, \mathrm{N}_{\text {hops }}=8, X=4$

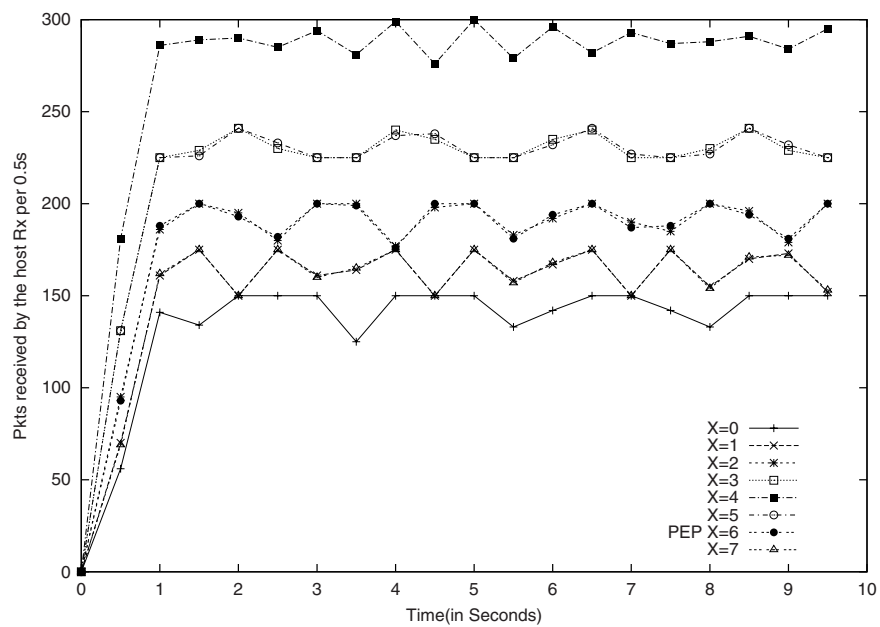

Fig. 5. Effect of PTS Node Location on TCP Throughput. $L_{C}=10 M b, L_{d}=$ $5 \mathrm{~ms}, T_{s}=100 \mu \mathrm{s}, N_{\text {hops }}=8, C_{\max }=1024 p k t s$

transmission on the outgoing link. Also in presence of multiple sessions competing for the CPU resources on the PTS node, a packet may have to wait longer until it gets serviced. Together this delay significantly affects the end-to-end performance of the PTS session. Figure 6 shows the effect of mean PTS service time on throughput. To represent a more realistic scenario, we used exponential distribution of $T_{s}$. When $T_{s}$ is very much less than rtt on PTS Connection2, PTS session will give better throughput than a direct TCP connection. It is important for PTS nodes to advertise their current estimate of service times so 


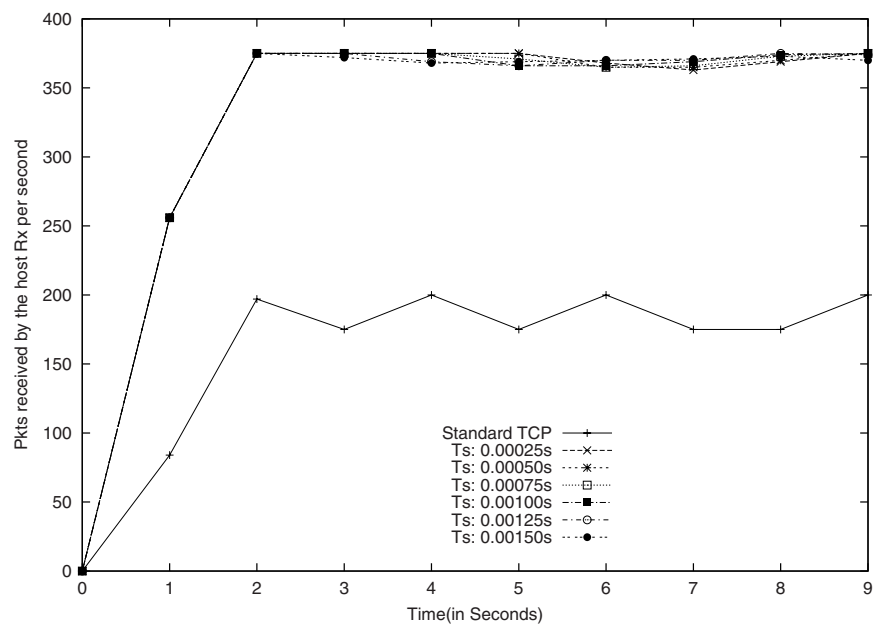

Fig. 6. Effect of PTS Delay on TCP Throughput. $L_{C}=10 M b, L_{d}=8 m s, N_{\text {hops }}=8$, $X=4, C_{\max }=400 p k t s$

that a host can choose a less loaded PTS node thereby achieving load sharing. Multiple PTS Nodes: In Figure 7 the two hosts are separated by distance of 14 hops. PTS node equal to 0 represents the throughput when a direct TCP connection is setup between the two hosts. As observed for one and two PTS nodes the performance is almost equal. As the number of PTS nodes increases beyond two, the throughput starts decreasing. Each TCP segment suffers a bounded amount of delay in every PTS node. This delay adds up to a significant amount if more than one PTS node is used, thus a single PTS node is adequate for proxy transport service.

\section{Proxy Transport Service (PTS)}

PTS complements TCP and is a nontransparent service that employs active nodes for code execution within the network. In this paper, we are proposing PTS as one of the predeployed services available to the end user as well as to other active network services. In PTS, predeployed programs or services can be checked to have security loop holes and they can be fine-tuned for high performance. Unlike schemes in [5]6], PTS is an on-demand service i.e., a host must explicitly make a request for PTS service to the active node. In order for an end host to use PTS, a PTS clients must reside on the source as well as the destination. The PTS component inside an active node is referred to as PTS. Figure 8 shows the interaction between the end systems and the active node components for setting up a PTS session.

The PTS Client is responsible for initiating a PTS connection setup request, selecting a PTS node for a session and, establishing and terminating a PTS connection. The PTS Client hides these details from the application. PTS Clients 


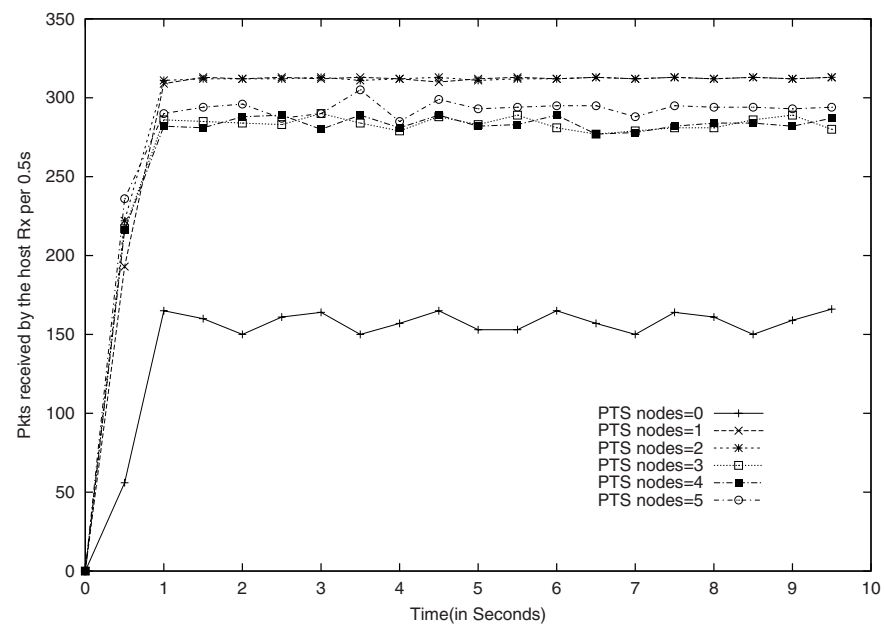

Fig. 7. Multiple PTS Nodes. $L_{C}=5 M b, L_{d}=2 m s, N_{\text {hops }}=14, T_{s}=1 \mathrm{~ms}, C_{\max }=$ 1024 pkts

communicate with the PTS through active packets using unused IP header bits to identify an active packet [4,9]. When this active packet arrives at a passive node it is routed using information in its IP header via the fast forwarding path. When an active packet arrives at an active node, the IP classifier sends it to the active engine for processing. In addition to rtt, TCP throughput also depends on the bandwidth of the connection. The location of the PTS node determines the end-to-end performance of PTS. Ideally, a PTS node should be located approximately in the middle of the path connecting the two hosts so that, $r t t_{(P T S \text { Connection-1) }}=r t t_{(P T S \text { Connection-2) }}=\frac{1}{2} r t t_{\text {total }}$. A requesting host may not be able to find an active node or PTS service at a location which can give optimal performance, settling for suboptimal but "better than direct TCP connection" performance. Therefore the PTS node should be chosen in such a way that the difference between the rtt's of PTS Connection-1 and PTSConnection-2 is minimal.

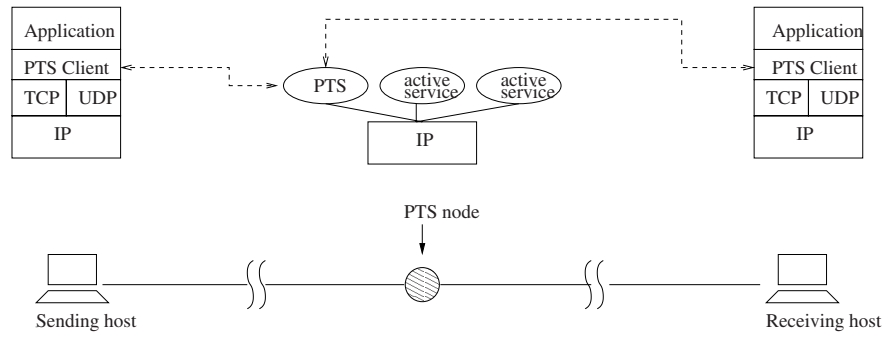

Fig. 8. PTS Architecture 
PTS Node Discovery: It is the mechanism by which PTS nodes are discovered and their location and status conveyed to the hosts. Using the knowledge obtained from PTS node discovery, the initiating host evaluates the suitability of a PTS node for its application. This mechanism is called PTS node selection. Since PTS is a host initiated service, a host must contact a PTS node requesting for proxy service. Therefore an initiating host must be aware of all the PTS capable active nodes located in the path (between the sender and the receiver). Also the initiating host needs information about the status of the PTS node, specifically: the location of the PTS node in terms of $r t t\left(r t t_{X}\right)$, current PTS service time estimate $\left(T_{s}\right)$ of the PTS node and, maximum buffer capacity $\left(C_{\text {max }}\right)$ that can be provided by the PTS node for this session. This information is used to evaluate a rank expression for each PTS node. Higher the value of the rank expression, better is the suitability of the PTS node in consideration. A basic PTS node discovery mechanism is as follows: 1) The initiating or source host sends an active, PTS probe datagram (including timestamp) to the destination host, 2)A passive node in the path will forward this datagram on the fast forwarding path, whereas an active node, in addition to forwarding the datagram to the destination host, will also send a copy to its active engine. If the PTS service is available, a PTS Available packet is generated which advertises this active node as a PTS node to the initiating host. The timestamp in the PTS probe packet is echoed back in the PTS Available packet so that the initiating host can calculate the $r t t$ between itself and the PTS node. This packet also contains information about the current state (PTS service time per average size packet and maximum buffer space) of the service on this active node. If the PTS service is not available on this node, the active engine ignores this datagram. A PTS node may not send any more PTS Available packets if it is already overloaded with PTS sessions. Finally when the destination host receives a PTS probe packet, it sends back an acknowledgment to the sending host echoing back the timestamp in the PTS probe packet. This helps the host to estimate the total rtt. 3) The initiating host collects all the replies to the PTS probe message. If the only PTS Available message received is from the destination host then it means that there are no active nodes hosting PTS service currently available along the path between the sender and the receiver. In this case, a direct TCP connection is established between the sender and the receiver. If no reply is received from the destination host within a certain period of time, the sender times out and retransmits the PTS probe datagram, discarding all the PTS available messages received if any. Setup Time Comparison with direct TCP: The setup time for a direct TCP connection is given by $t_{\text {setup }} \approx \frac{3}{2} r t t_{\text {total }}$. We can deduce the setup time $t_{\text {setup }}$ for a PTS session as, $t_{\text {setup }} \approx r t t_{\text {total }}+\frac{1}{2} r t t_{X}+t_{s}+\left(r t t_{\text {total }}-r t t_{X}\right)+r t t_{X} \approx$ $2 r t t_{\text {total }}+\frac{1}{2} r t t_{X}+t_{s}$ where $t_{s}$ is the time required by the PTS node to service the connection setup request. If only one PTS available message is received (from the destination host) then a direct TCP connection will have to be setup because there is no PTS node along the path. In this case the setup time is, $t_{\text {setup }} \prime \prime \approx \frac{5}{2} r t t_{\text {total }}$. As seen from the above equations, $t_{\text {setup }}$ as well as $t_{\text {setup }} \prime \prime$ are greater than $t_{\text {setup. }}$. The PTS node discovery takes about one $r t t_{\text {total }}$ more 
than the time required to setup a direct TCP connection. If any intermediate router drops the PTS probe packet then it will take more than one $r_{t} t_{\text {total }}$ to discover PTS nodes. For this reason, use of PTS for short sessions(small data transfer) is not justified if the primitive approach is used to discover PTS nodes. Instead of the host discovering PTS nodes when there is a request for PTS service, a more proactive approach can be adopted in which PTS nodes are probed periodically.

Proactive PTS Node Discovery: In order to discover PTS nodes proactively every host can send PTS probe messages periodically to its prospective destinations and build a table of services available along that path. But there are problems with this naive approach. If every host in the network is going to periodically perform PTS node discovery like this, then the network will be inundated with the 'probe' and 'reply' messages. Also it is difficult to guess in advance the destinations with which the host will be communicating. Another problem lies in the timeliness of the PTS service information being gathered. The services table may not reflect the most up to date status of the PTS node e.g., between the probing interval the loads might change or the routes itself might change making a particular PTS node no longer appear on the route to a destination. There are additional problems if the end point is not a conventional computing element. If the initiating host is a wireless device, PDA or mobile phone for example, then it may not have sufficient memory to store the services table, nor is it advisable to perform PTS node discovery over the already bandwidth constrained and unreliable wireless link. PTS node discovery can be accomplished by deploying an active service offered over active networks.

\section{Conclusions}

Proxy transport service (PTS) proposed in this paper is an active network based service targeted towards improving the TCP throughput of large $r t t$ connections. PTS results in faster rate of increase of the source host's congestion window leading to higher TCP throughput. The main difference between PTS and other enhancing schemes is the non-transparent nature of PTS service. PTS is not localized to any node and can be deployed on any node within the network. As active nodes proliferate the network, higher is the probability that a host will find a suitable PTS node, leading to better load sharing among the active nodes providing PTS service. We have demonstrated the working of PTS and proved its advantages over a direct TCP connection for large rtt connections. Three major factors affecting the PTS throughput are: PTS node location, PTS service time per packet and PTS buffer size per session. With the availability of high speed processors, efficient active node architectures, and distributed nature of PTS, PTS service can scale to large number of users. PTS node discovery can be accomplished by agents executing on active nodes. A proactive PTS Node discovery scheme can reduce the setup time. 
Acknowledgements. This material is based upon work supported by the National Science Foundation under Grant No. 0129682.

\section{References}

1. Tennenhouse, D.L., Smith, J.M., Sincoskie, W.D., Wetherall, D.J., Minden, G.J.: A Survey of Active Network Research. IEEE Communications Magazine 35 (1997) $80-86$

2. Tennenhouse, D.L., Wetherall, D.J.: Towards an Active Network Architecture. Computer Communication Review 26 (1996)

3. Wetherall, D., Legedza, U., Guttag, J.: Introducing new internet services: Why and how. IEEE Network Magazine, special issue on Active and Programmable Networks (1998)

4. Psounis, K.: Active Networks: Applications, Security, Safety, and Architectures. IEEE Communications Surveys (1999) 1-16

5. Bakre, A., Badrinath, B.R.: I-TCP: Indirect TCP for mobile hosts. 15th International Conference on Distributed Computing Systems (1995)

6. Dutta, D., Zhang, Y.: An Active Proxy Based Architecture for TCP in Heterogenous Variable Bandwidth Networks. IEEE GLOBECOM (2001)

7. Zhang, L.: Resource Reservation Protocol-Version 1 Functional Specification. Network Working Group -RFC 2205 (1997)

8. Breslau, L., Estrin, D., Fall, K., Floyd, S., Heidemann, J., Helmy, A., Huang, P., McCanne, S., Varadhan, K., Xu, Y., Yu, H.: Advances in Network Simulation. IEEE Computer 33 (2000) 59-67

9. Calvert, K.: Directions in Active networks. IEEE Communications Magazine: Special Issue on Programmable Networks (1998) 